\title{
More than one way to induce a neuron
}

\begin{abstract}
Seventy-six pairs of transcription factors can induce mouse connective-tissue cells to adopt a neuron-like identity in vitro. This discovery provides insights into both neuronal development and cell reprogramming. SEE ARTICLE P.375
\end{abstract}

\section{LYNETTE LIM \& OSCAR MARIN}

$\mathrm{T}$ The brain contains hundreds of neuronal subtypes, each defined by a specific combination of features, including its position and shape, the neurotransmitter molecules it produces and its electrophysiological properties ${ }^{1}$. Engineering this enormous diversity in the laboratory is an ultimate goal of regenerative medicine. On page 375 , Tsunemoto et al. ${ }^{2}$ describe a large-scale effort to identify factors that can endow non-neural cells cultured in vitro with neuronal properties.

Understanding the mechanisms that underlie the generation of neuronal diversity has been a central goal of neurobiology for more than a hundred years, since the neuroscientist Santiago Ramón y Cajal postulated that the nervous system is made up of discrete individual cells ${ }^{3}$. Work over the past three decades has identified gene regulatory networks that control neuronal identity as it unfolds in the embryonic brain ${ }^{4}$. These studies have also revealed that neuronal identity is intimately linked to the environment in which neurons develop, primarily because some of the cells' most important features, such as their connections, depend on their interactions with other neurons.
In the past decade, however, it has become clear that many neuronal attributes can be generated outside the normal context of brain development. For example, in 2010 it emerged $^{5}$ that a cocktail of three transcription factors can be applied to fibroblasts (the most common cells of connective tissue) cultured in vitro to convert them into cells that resemble brainderived neurons, at least in terms of their shape and electrophysiological properties. This procedure, called direct lineage reprogramming, is based on the premise that certain transcription factors regulate gene-expression patterns characteristic of neuronal cell types. But what has not been clear is whether the capacity to reprogram cells into neurons is limited to a handful of transcription factors.

Previous work by the group that performed the current study showed that a pair of transcription factors from the basic helix-loophelix (bHLH) and Pit-Oct-Unc (POU) families can induce the expression of neuronal markers through direct reprogramming ${ }^{6}$. Tsunemoto et al. were inspired by this finding in their current work. The authors screened 598 pairs of bHLH and POU transcription factors chosen on the basis of their expression in neuronal lineages - to see which could transform
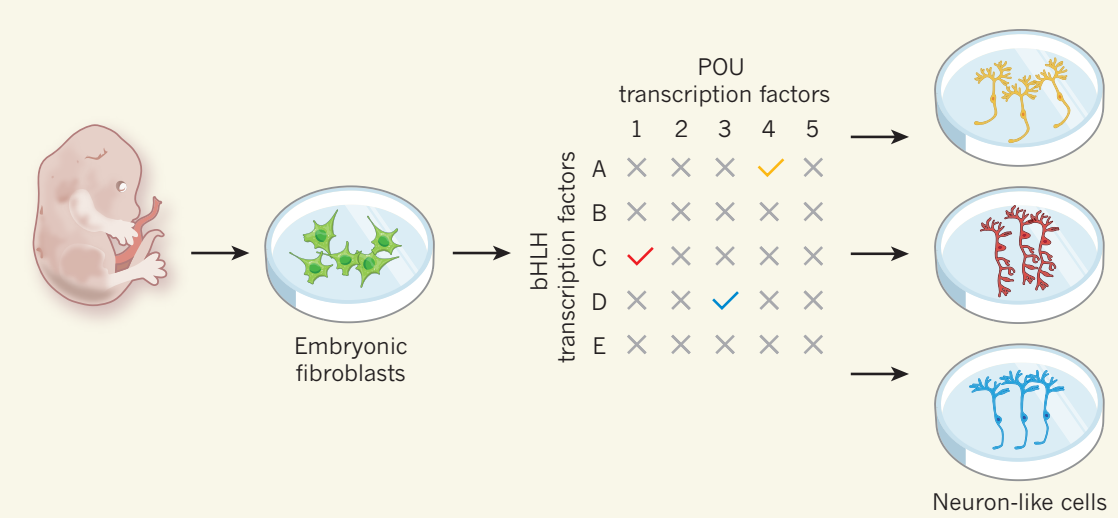

Figure 1 | Inducing neurons through direct reprogramming. Tsunemoto et al. ${ }^{2}$ grew embryonic fibroblasts (connective-tissue cells isolated from mouse embryos) in vitro. They treated the cells with 598 pairs of transcription factors from the basic helix-loop-helix (bHLH) and Pit-Oct-Unc (POU) families, most of which are expressed in neuronal lineages. In total, more than $12 \%$ of the transcriptionfactor combinations tested could reprogram the fibroblasts into cells with neuronal properties (indicated with ticks). Different pairs produced neuron-like cells with different shapes, gene-expression profiles and electrophysiological properties (indicated by different colours). mouse embryonic fibroblasts into neurons in vitro (Fig. 1). Seventy-six of the pairs produced cells that expressed multiple markers of mature neurons and had neuronal morphologies. Thus, neuronal features can be induced in non-neuronal cells by an astonishing range of transcription-factor combinations.

How similar are the neurons induced by the transcription-factor pairs? Analysis of gene expression using single-cell RNA sequencing revealed that a given pair of factors generates relatively homogeneous populations of neurons, which share a similar molecular profile. This is surprising, because previous experiments have highlighted the heterogeneity of cell populations undergoing reprogramming in culture ${ }^{7}$.

By contrast, Tsunemoto et al. found that different transcription-factor combinations induced the formation of distinct neuronlike populations that had characteristic markers and electrophysiological features. However, the authors also found that certain features - such as the expression of particular ion channels or neurotransmitter receptors could be induced by multiple combinations of transcription factors. These findings support the idea that there is not a single 'gene code' for making a particular feature of neuronal identity, but that the molecular machinery underlying neuronal development has a remarkable degree of redundancy. In other words, identity-defining transcription factors are probably used in different combinations in distinct neuronal cell types to generate the diversity seen in vivo.

One key question is whether the induced neurons faithfully mimic neuronal cell types found in vivo, or whether (and to what extent) they represent artificial cell types. To address this issue, the authors compared gene-expression patterns in induced cell populations with those in neuronal subtypes taken from threeweek-old mice. This analysis indicated that the induced neurons did not match endogenous cell types of the juvenile mouse brain. However, it might be that the induced neurons did not reach the same stage of development as endogenous cells. Matching cell types across different developmental stages remains a complex challenge in neurobiology, as shown this year by two RNA-sequencing studies ${ }^{8,9}$. These highlighted the difficulty of recognizing the transcription factors that define specific cell types at early stages of neuronal development in the brain's cerebral cortex.

It is possible, then, that the neurons induced in vitro by Tsunemoto et al. correspond to specific populations of endogenous neurons in a relatively immature state. These cells might develop into fully differentiated neurons only if placed in the appropriate environment. Alternatively, the expression of a pair of transcription factors might be sufficient to elicit the development of some neuronal features in fibroblasts, but not to unleash the complete program of 
differentiation that takes place in the embryo.

Nevertheless, Tsunemoto and colleagues' study adds to the body of evidence showing that some features of neuronal identity can be reproduced outside the developing brain. In doing so, it demonstrates the power of reprogramming to interrogate the function of neuron-specific genes. The authors have made their findings available in a database (see go.nature.com $/ 2 \mathrm{r} 1 \mathrm{msxi}$ ) that will allow other researchers to use the transcription-factor codes to induce specific neuronal features. This will doubtless prove useful for studying the selective vulnerability of specific neuronal subtypes to disease.
Finally, the authors provide preliminary evidence that their transcription-factor combinations can also be used to generate neurons from human embryonic fibroblast-like cells. Following further validation, the codes might help us to decipher the origins of neuronal diversity in humans.

\section{Lynette Lim and Oscar Marín are in the} Centre for Developmental Neurobiology, Institute of Psychiatry, Psychology and Neuroscience, King's College London, London SE1 1UL, UK, and in the MRC Centre for Neurodevelopmental Disorders, King's College London. e-mail:oscar.marin@kcl.ac.uk

1. Zeng, H. \& Sanes, J. R. Nature Rev. Neurosci. 18, 530-546 (2017).

2. Tsunemoto, R. et al. Nature 557, 375-380 (2018).

3. Ramón y Cajal, S. Histologie du Système Nerveux de l'Homme et des Vertébrés (Maloine, 1909).

4. Lodato, S. \& Arlotta, P. Annu. Rev. Cell. Dev. Biol. 31 699-720 (2015).

5. Vierbuchen, T. et al. Nature $463,1035-1041$ (2010).

6. Blanchard, J. W. et al. Nature Neurosci. 18, 25-35 (2015).

7. Treutlein, B. et al. Nature 534, 391-395 (2016).

8. Mi, D. et al. Science 360, 81-85 (2018).

9. Mayer, C. et al. Nature 555, 457-462 (2018).

This article was published online on 9 May 2018.

\section{Increased emissions of ozone depleters}

Chlorofluorocarbons are the main class of chemical that depleted the ozone layer in the stratosphere. Measurements reveal that emissions of these compounds are rising again, despite international rules restricting their use. SEE LETTER P.413

\section{MICHAELA I. HEGGLIN}

$\mathrm{M}$ onitoring the expected decline in the atmospheric concentrations of banned compounds might seem like an unexciting research task. But on page 413, Montzka et al. ${ }^{1}$ report an unexpected finding in the long-term measurements of CFC-11, one of the most potent ozone-depleting compounds: its atmospheric concentration is decreasing much more slowly than would be expected on the This points to a fresh rise in emissions - in contravention of international regulations.

CFC-11 belongs to the chlorofluorocarbon (CFC) family of compounds. CFCs are highly stable, synthetic chemicals that were used in various applications from the 1930s onwards - for example, as propellants in aerosol sprays, solvents and refrigerants. In the early 1970s, the British chemist James Lovelock and his colleagues were the first to measure the abundance of CFCs in the atmosphere and to realize that these substances were found ubiquitously in both the Northern and Southern hemispheres, despite their sources being located only in the Northern Hemisphere ${ }^{2}$. This finding led to the hypothesis that CFCs could be destroyed naturally only in the stratosphere, in a process that releases chlorine atoms. Each of basis of its known sources and sinks. these atoms would be able to destroy many ozone molecules in catalytically driven cycles, thus posing a threat to the ozone layer ${ }^{3}$, which protects life on Earth from harmful ultraviolet radiation.

The discovery ${ }^{4}$ of the 'hole' in the ozone layer over Antarctica in 1985 proved this hypothesis to be not only correct, but also much more threatening than had been imagined. It spurred research activities to understand why such severe ozone depletion was found over Antarctica alone, and led to political action to restrict the use of CFCs under the Montreal Protocol in 1987. The realization that more-severe ozone depletion would spread further across the globe if CFCs continued to be released into the atmosphere, along with technological advances that made the replacement of CFCs possible, helped governments to tighten the regulations on CFCs and ultimately ban their production through several amendments to the protocol. As a result of these actions, CFC concentrations in the atmosphere peaked in the mid-to-late 1990s and have been steadily declining ever since ${ }^{5}$. The Montreal Protocol has been hailed as the most successful international treaty so far that deals with a global environmental issue ${ }^{6}$.

Because the destruction of CFCs in the stratosphere is a slow process, their removal from the atmosphere will take many decades. Today's research into stratospheric ozone focuses on whether atmospheric concentrations of ozone-depleting substances are decreasing as expected, and whether the ozone layer is on its way to recovery. Working out whether the ozone layer is recovering on the basis of ozone observations alone is particularly difficult because of confounding effects from natural variability, climate change and ozone pollution ${ }^{7}$.

Monitoring the atmospheric concentration of ozone-depleting substances such as CFC-11 is a more direct test of the effectiveness of the Montreal Protocol. However, even for these long-lived chemicals, natural variability in the transport of air masses between the source and sink regions of the chemicals can affect the rate of the expected decline. The source regions are mostly found in 